\title{
Obstrucción infravesical tras cabestrillo tipo Remeex
}

\author{
Bujons Tur A, Errando Smet C, Prados Saavedra M*, Báez C, Arañó Bertrán P, \\ Gutiérrez Ruiz C, Villavicencio Mavrich H. \\ Unidad de urología funcional y femenina. Servicio de Urología Fundació Puigvert. Barcelona. \\ *Servicio de Urología Hospital Juan XXIII Tarragona. \\ Actas Urol Esp. 2006;31(1):43-48
}

\section{RESUMEN}

\section{OBSTRUCCIÓN INFRAVESICAL TRAS CABESTRILLO TIPO REMEEX}

La colocación de un cabestrillo de tensión regulable tipo Remeex, es un procedimiento quirúrgico destinado a corregir la incontinencia urinaria femenina. Contiene un varitensor que regula la tensión del sistema sobre la uretra, elemento central de la prótesis que quedará en la paciente y un manipulador, elemento que actúa sobre el varitensor y modificar la tensión bajo la uretra en el postoperatorio inmediato o incluso tiempo después.

Presentamos el caso clínico de una paciente diagnosticada de incontinencia urinaria de esfuerzo y el tratamiento quirúrgico realizado con sus ventajas respecto a otros sistemas de corrección antiincontinencia que se realizan en la actualidad.

Palabras clave: Incontinencia de orina. Cabestrillo Remeex. Obstrucción.

\section{ABSTRACT \\ LOW OBSTRUCTION TRACT BY SYSTEM REEMEX}

The Remeex system it's a sling with regulable tension created for the surgical correction of the female urinary incontinente. The system contains a varitensor central parto $f$ the protesis that regulates the tension of the uretra. The screwdriver that acts against the varitensor to the modify the tension under the uretra in the postop inmediately or time after also. We present a clinic case of a patient with diagnostic of urinary stress incontinence and the surgical treatment with advantages against others kind of systems used for incontinence treatment at this time.

Keywords: Stress incontinence. Remeex system. Obstruction.

$\mathrm{P}$ resentamos el caso clínico de una paciente diagnosticada de incontinencia urinaria de esfuerzo y el tratamiento quirúrgico realizado con sus ventajas respecto a otros sistemas de corrección antiincontinencia que se realizan en la actualidad.

\section{CASO CLÍNICO}

Mujer de 53 años de edad, sin antecedentes patológicos médicos ni quirúrgicos de interés, consulta a nuestro centro por presentar incontinencia urinaria a moderados esfuerzos (toser, correr ...) de años de evolución, con algún escape en reposo y sin referir imperiosidad miccional.

Como antecedentes ginecológicos refiere 2 partos eutócicos previos.

En la Exploración vaginal no se objetivan masas ni celes, evidenciando importante escape con la tos durante la exploración. Qtip test negativo $\left(20^{\circ}\right)$.

Como exploraciones complementarias se realiza un estudio urodinámico para valorar la incontinencia de orina (Cistomanometría y estudio de presión-flujo) y perfil uretral (Figs. 1, 2, y 3). 


\section{Cistometría}

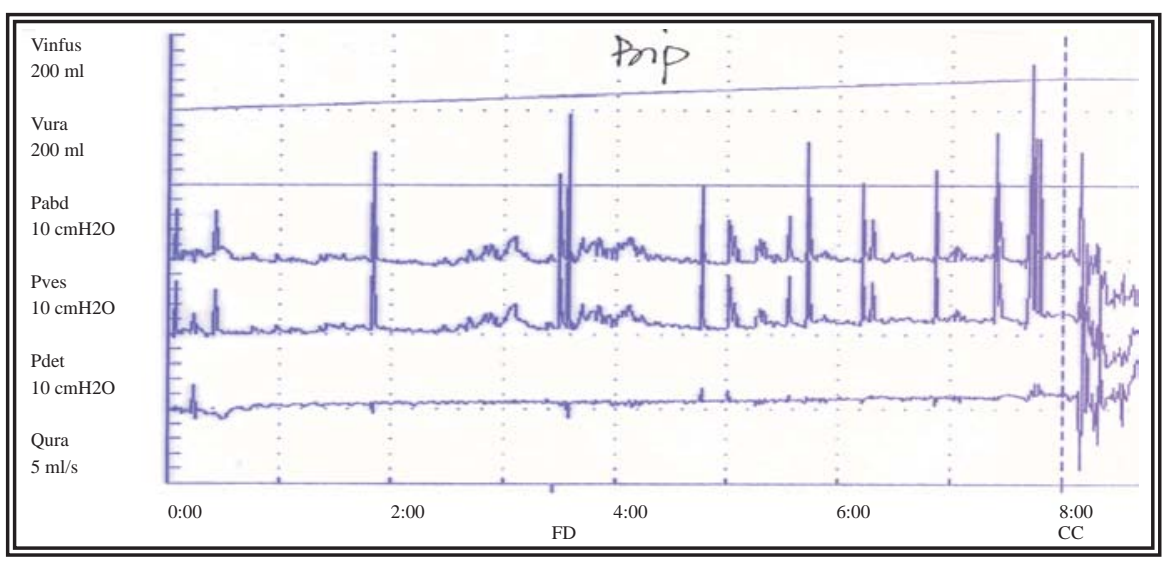

FIGURA 1. Detrusor estable. Ausencia de escape con tos.

Presión-Flujo

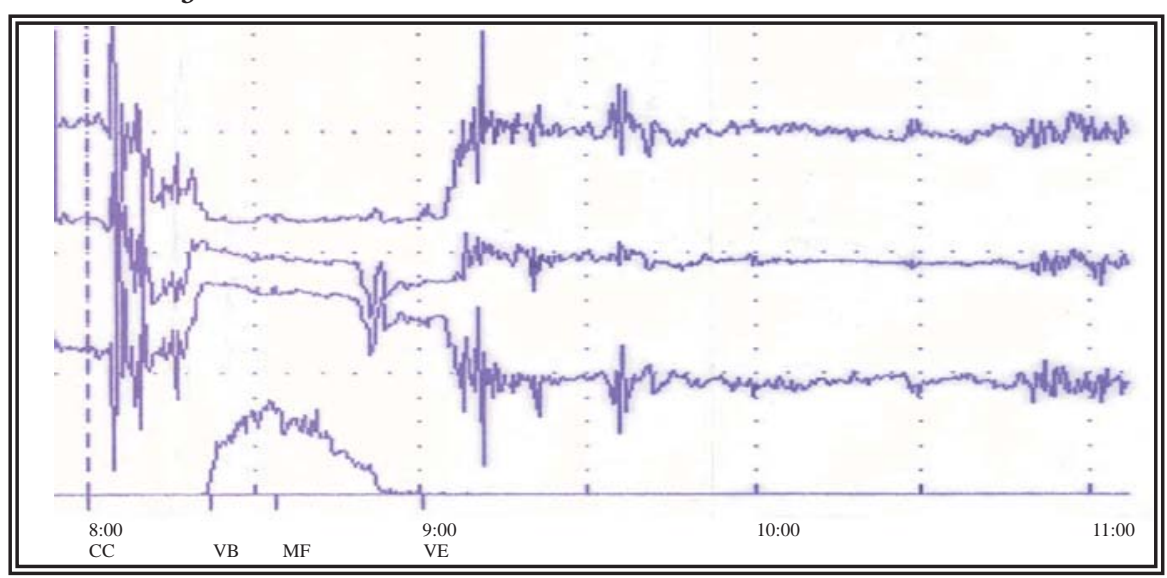

FIGURA 2. Contracción sostenida del detrusor. Se descarta obstrucción infravesical.
Con el diagnóstico de insuficiencia esfinteriana por clínica, no contraindica cirugía reparadora de incontinencia decidiéndose intervención quirúrgica.

Dadas las características clínicas (Incontinencia de orina de esfuerzo grado III) y anatómicas de la paciente se decide colocación de un cabestrillo de tensión regulable tipo Remeex sin incidencias. A las 24 horas de la intervención quirúrgica se retira sonda vesical con diuresis espontánea y sin residuo postmiccional. A las 48 horas se inicia la regulación del sistema; para ello, se introducen 300 cc de suero fisiológico en el interior de la vejiga y se realiza la prueba de la tos con resultado negativo, un Pad test negativo pero con residuo postmiccional considerable. Siguiendo el esquema terapéutico, con la sospecha de exceso de tensión del sistema bajo la uretra se procede a la destensión girando

Perfil uretral

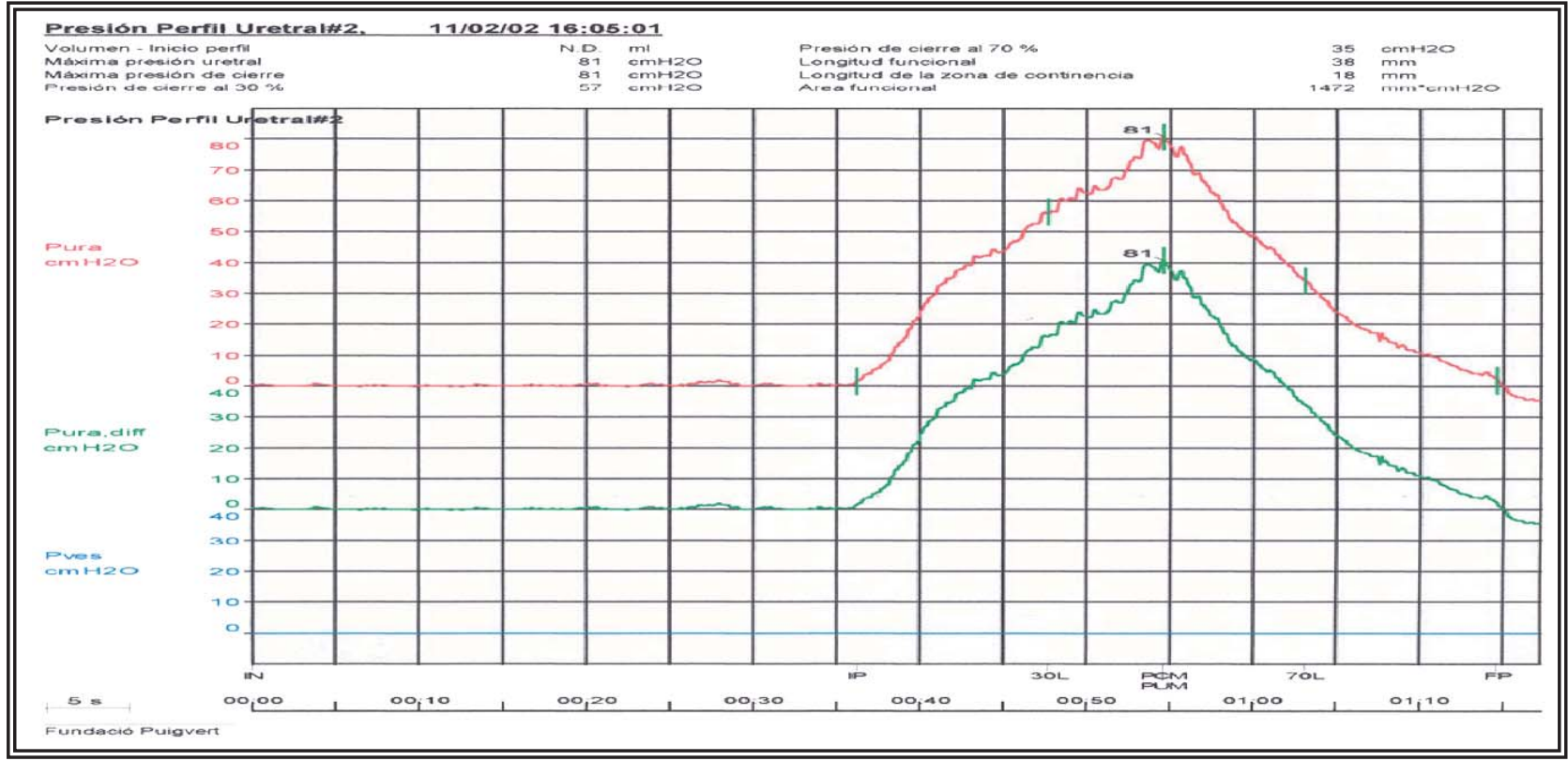

FIGURA 3. Perfil uretral normal. 
4 vueltas el manipulador en sentido antihorario y basculación uretral posterior con comprobación de la desaparición del residuo. Finalmente se realizan dos Pad test con resultado negativo y se retira el manipulador del varitensor siendo dada de alta la paciente.

Al mes de la cirugía, acude a la consulta por referir sintomatología clínica miccional mixta: chorro fino, entrecortado, dolor hipogástrico, sensación de vaciado incompleto, prensa abdominal, polaquiuria y escapes por imperiosidad.

En la Exploración física no se objetivan escapes con la tos, ni presencia de fístulas o erosión

\section{Flujometría}

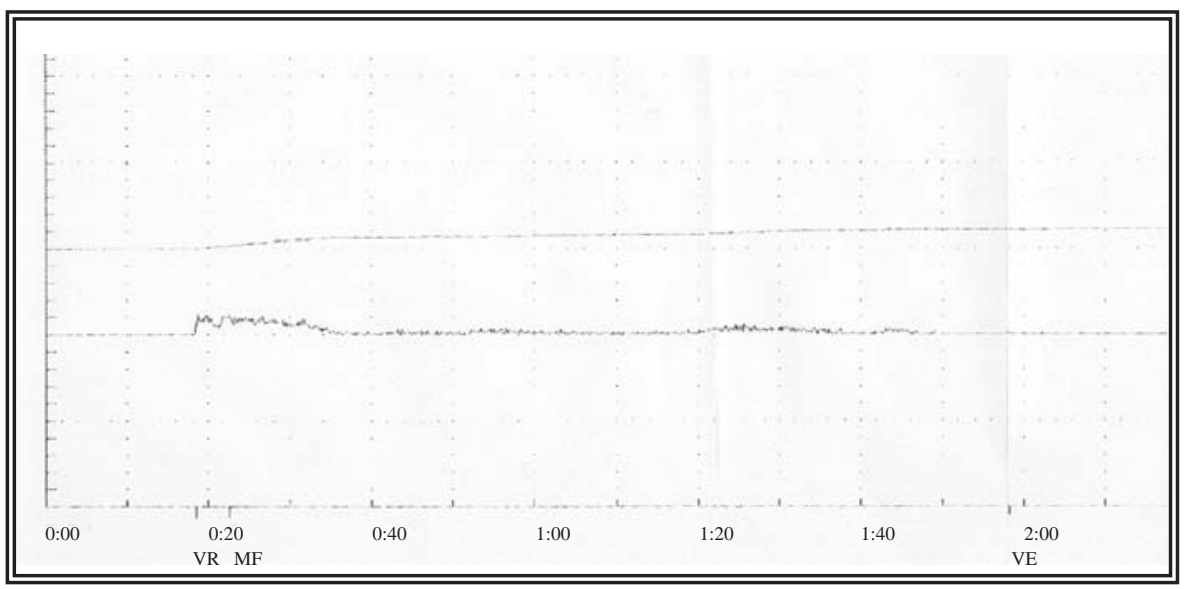

FIGURA 4. Flujometría con $Q$ máx. 5.7ml/s, Vol. $104 \mathrm{ml}$ y RPM $412 \mathrm{ml}$. de la malla en vagina. Se realiza análisis de orina con resultado negativo y para descartar posible fístula, cistoscopia comprobando ausencia de malla en vejiga o uretra ni fístula.

El estudio urodinámico nos informa de obstrucción infravesical (Figs. 4, 5 y 6).

Con el diagnostico urodinámico de obstrucción infravesical se implanta el manipulador al varitensor y se destensan 5 vueltas en sentido antihorario más basculación uretral posterior bajo anestesia local. Para comprobar si se ha desobstruido la paciente se realiza flujo fisiológico (Fig. 7) objetivándose persistencia de escaso caudal. Se destensan 15 vueltas más (para un total de 24 vueltas) con mejoría subjetiva del caudal miccional. Se comprueba con un flujo fisiológico con $\mathrm{Q}$. más $22 \mathrm{ml} / \mathrm{s}$ con $270 \mathrm{ml}$ de volumen, una prueba de la tos negativa, un Pad test negativo y escaso residuo postmiccional. Dada la mejoría clínica de la paciente con desaparición del escape y ausencia de residuo postmiccional se desconecta el manipulador (Fig. 8).

\section{Cistometría}

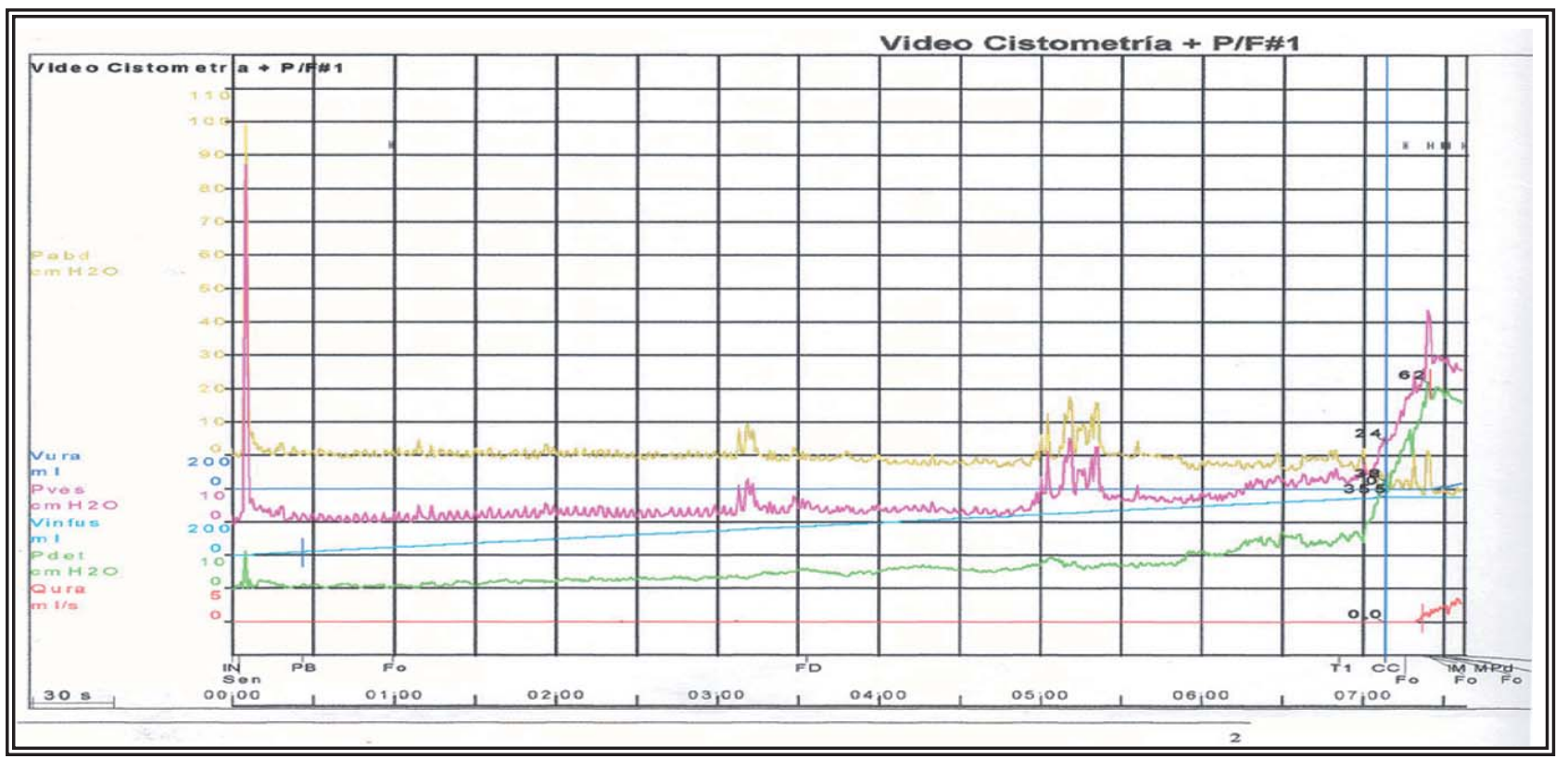

FIGURA 5. Detrusor estable. No escapes. 
Presión-flujo

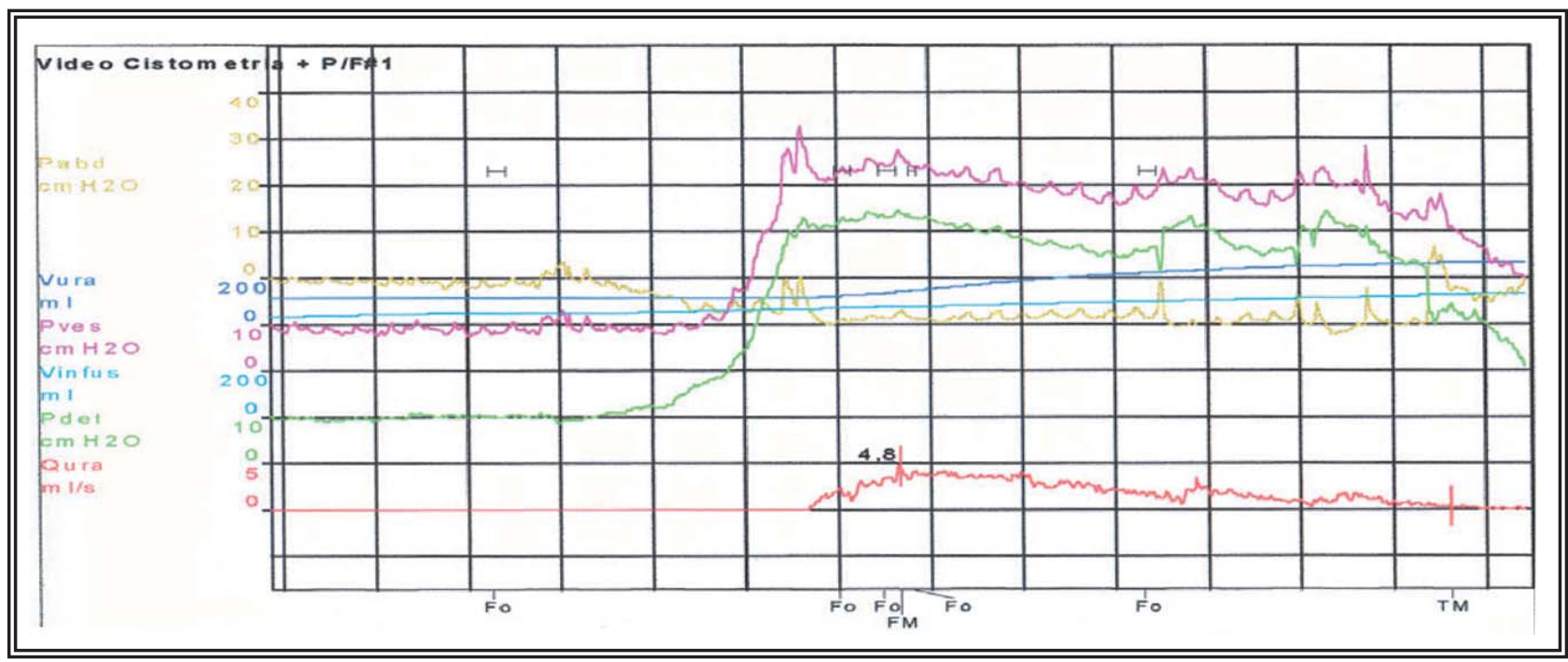

FIGURA 6. Pdet en Gmáx de 46cm de H2O. Qmáx 5ml/s, R.U 1.8cm H2O/ml/s, RPM 338ml

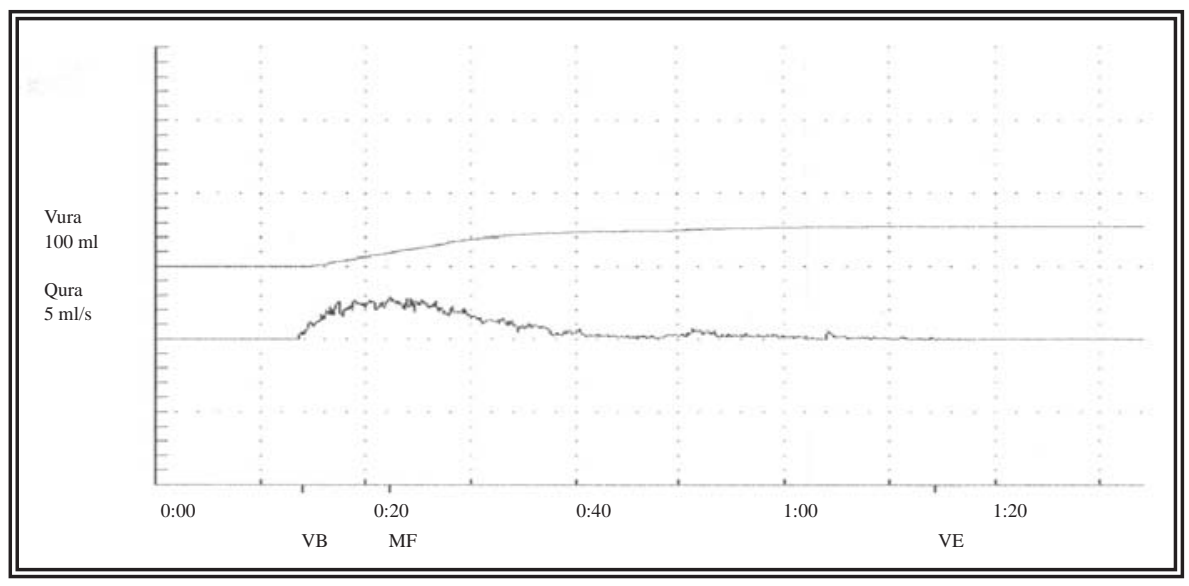

FIGURA 7. Flujometría fisiológica con Qmáx $14.6 \mathrm{ml} / \mathrm{s}$, volumen $247 \mathrm{ml}$, tras destensión de 5 vueltas.

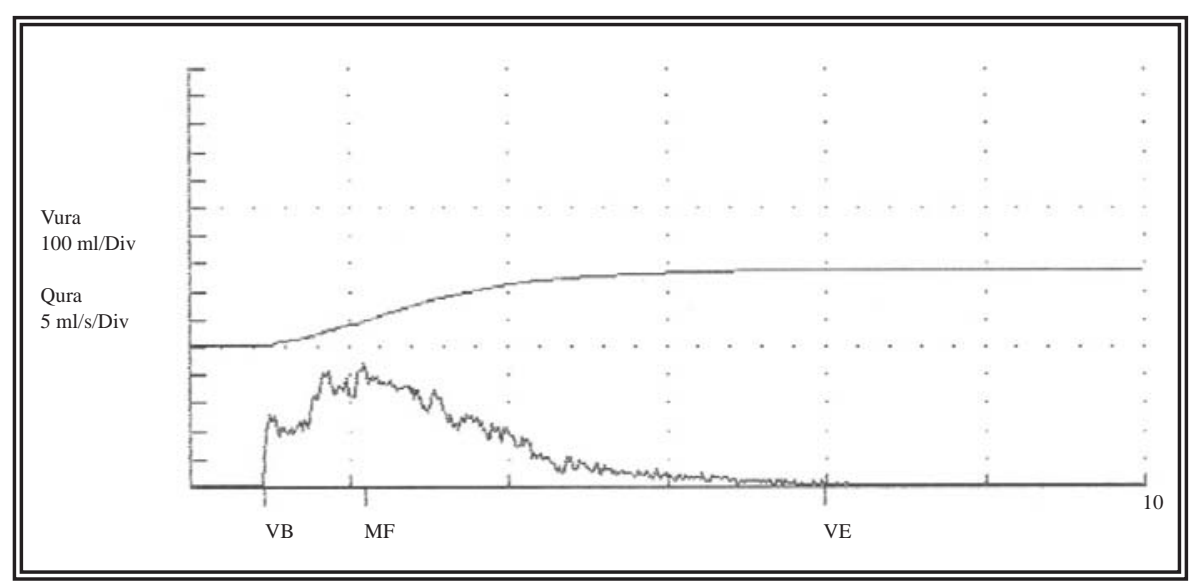

FIGURA 8. Flujometría fisiológica con Qmáx $22 \mathrm{ml} / \mathrm{s}$, volumen $276 \mathrm{ml}$ tras destensión de 15 vueltas más.

\section{DISCUSIŌN}

La colocación de un cabestrillo de tensión regulable tipo REMEEX, es un procedimiento quirúrgico destinado a corregir la incontinencia urinaria femenina. En nuestro centro las indicaciones de dicha técnica quirúrgica son: Incontinencia urinaria de esfuerzo grado III, incontinencia recidivada tras cirugía o con uretra fija.

El cabestrillo de tensión regulable tipo Remeex consta de los siguientes elementos:

- Varitensor: Regula la tensión del sistema sobre la uretra. Es el elemento central de la prótesis que quedará en la paciente. Consiste en un pequeño óvulo de Chirulen (Polietileno biocompatible de alta densidad) que contiene en su interior un carrete de ruedas dentadas de titanio que recogen los hilos de tracción (una vuelta completa eleva $1 \mathrm{~mm}$ el plano 
de suspensión) con la finalidad de aumentar o disminuir la tensión que el sistema ofrece sobre la uretra.

- Manipulador: Elemento que actúa sobre el varitensor para modificar la tensión bajo la uretra. Se une al varitensor por medio del desacoplador.

- Desacoplador: Elemento similar a un desatornillador que sirve para acoplar o desacoplar el manipulador al varitensor.

- Cabestrillo: Malla de monofilamento de polipropileno de $30 \times 1.25 \mathrm{~mm}$ unida a dos hilos de tensión de polipropileno que hará la función de soporte bajo la uretra.

- Pasador de los hilos de tracción: Aguja de 6 mm de diámetro, de curva y punta roma con dos agujeros pasahilos, para arrastrar los hilos de tracción desde el espacio vaginal a través del espacio de Retzius hasta atravesar la fascia de los músculos rectos.

- Mango de pasador de hilos: Mango acoplable al pasador de hilos de tracción que ayudan a dirigir el pasador de hilos.

\section{TECNICA QUIRÚRGICA}

Se coloca a la paciente en posición ginecológica/litotomía. Se realiza una incisión en longitudinal a nivel tercio medio uretral, posteriormente se realiza disección de los espacios parauretrales hasta el diafragma urogenital. Posteriormente se pasa al campo hipogástrico donde se realiza una incisión transversa de unos $3-5 \mathrm{~cm}$, una disección del tejido graso subcutáneo hasta exponer la fascia de los músculos rectos del abdomen. Se introducen los pasadores por el espacio parauretral a través del espacio de Retzius hacia el campo vaginal, a nivel de los vértices de la incisión realizada. Opcionalmente en pacientes obesas se puede acompañar el pasador con el mango correspondiente y movilizar la uretra con diferentes técnicas.

Posteriormente se realiza cistoscopia para comprobar indemnidad vesical durante el paso de las agujas.

Se coloca el cabestrillo en tercio medio de uretra. Se pasan los hilos de tracción a través de los orificios de las agujas. Se avanzan los pasadores de suturas arrastrando los hilos de tracción hasta que estos atraviesen la fascia de los mús- culos rectos del abdomen y alcanzar el campo abdominal. Se fija la malla a nivel periuretral para evitar el movimiento de ésta durante los ajustes.

Se coloca la prótesis a nivel campo abdominal, se pasan los hilos de tracción por el agujero lateral del varitensor (del mismo lado) hasta extraerlo por el orificio central. Una vez pasados los hilos de tracción el ayudante mantiene la prótesis Remeex a $10 \mathrm{~cm}$ de la fascia de los músculos rectos abdominales y sobre la línea media, anudamos los hilos de tracción del lado derecho sobre los del lado izquierdo con 5 nudos alternos. Se tiene que vigilar de tener la misma cantidad de hilo por cada lado y que éste ofrezca la misma tensión por cada lado.

Posteriormente se gira el manipulador en sentido horario para que los hilos de tracción se vayan enrollando en el eje del varitensor, hasta que la prótesis descanse sobre la fascia de los músculos rectos anteriores del abdomen. Cada vuelta completa recoge $1 \mathrm{~mm}$ de hilo dentro del varitensor.

Una de las ventajas que ofrece el sistema es la posibilidad de ajuste diferido del mismo tanto en caso de incontinencia postoperatoria como en caso de obstrucción tras la cirugía como es este caso.

La cirugía de la incontinencia es la causa más frecuente de obstrucción infravesical en la mujer. Entre un $2-24 \%$ de los casos operados de incontinencia urinaria de esfuerzo presentan una disfunción miccional de tipo obstructivo, irritativo o mixto. La retención urinaria, el residuo postmiccional y la prensa abdominal sugieren un componente obstructivo.

Las técnicas de "sling sin tensión" deradas actualmente como la técnica de elección en la incontinencia urinaria de esfuerzo femenina.

Los criterios diagnósticos de la obstrucción infravesical en la mujer no están bien definidos utilizándose: flujo bajo inf. $12 \mathrm{ml} / \mathrm{s}$, Pdet superior a $20 \mathrm{~cm} \mathrm{de} \mathrm{H}_{2} \mathrm{O}$, resistencia uretral $>0,2$.

Cuando el procedimiento quirúrgico es obstructivo se produce una disminución del flujo máximo. El residuo urinario es otro dato urodinámico importante a considerar. La combinación de presión detrusoriana a flujo máximo y presencia de residuo urinario puede dar lugar a un 
nomograma para diagnosticar una obstrucción urinaria femenina.

La relación temporal entre la intervención antiincontinencia y la aparición de la sintomatología es un criterio clínico de sospecha de obstrucción tras la cirugía.

La uretrolisis es una técnica efectiva en conseguir la desaparición de la sintomatología producida tras la obstrucción por cirugía antiincontinencia. Existen diferentes vías de abordaje de la uretrolisis dependiendo de la cirugía previa realizada $^{2}$.

La vía de abordaje para la cervicouretrolisis puede ser retropúbica, vaginal o suprameática.

Cuando la técnica utilizada ha sido un sling por vía vaginal, la simple sección de una de las ramas del sling parece ser suficiente para mejorar la clínica. Cuando ha sido por vía retropúbica, se realiza una liberación cervicouretral de las adherencias existentes. Posteriormente puede realizarse una nueva resuspensión uretral durante el mismo acto o diferida.

Posteriormente según la literatura, puede haber una desaparición prácticamente total de los síntomas en un $67 \%$ e los casos y una mejoría en un $33 \%$, con tasa de éxitos de $65-95 \% \%^{3,4}$. Refieren mejoría o desaparición de la urgencia con incontinencia tras la uretrolisis, desaparición o mejoría del residuo miccional, aunque en algunos casos puede persistir la incontinencia de esfuerzo que puede resolverse mediante la realización de una resuspensión cervicouretral, aunque es controvertido, porque si no se realiza existe la posibilidad de incontinencia urinaria de esfuerzo posterior en un $17 \%^{5}$ y si se realiza y persiste la obstrucción es dificil poder diferenciar la existencia de una nueva obstrucción si persisten los síntomas.

\section{CONCLUSIÓN}

Una de las ventajas que ofrece el cabestrillo de tensión regulable tipo Remeex es la mayor facilidad de resolver una de las complicaciones más temidas tras la colocación de cabestrillos como es la obstrucción. Clásicamente la obstrucción generada tras un cabestrillo se resuelve mediante la realización de uretrolisis.

Por el contrario en el cabestrillo Remeex se aborda la obstrucción con el implante del manipulador sobre el varitensor, bajo anestesia local. Técnica de fácil realización y que permite una regulación progresiva con control de la continencia.

\section{REFERENCIAS}

1. Romero Maroto J, Prieto Chaparro L, López López C, Quilez Fenoll JM, Rodriguez Fernandez E. Obstruction following surgical repair of female stress urinary incontinence. Diagnosis and treatment. Arch EspUrol;2002. 55(9):11071114.

2. Kuo HC. The surgical results of the pubovaginal sling procedure using polypropylene mesh for stress urinary incontinence. BJU International; 200188(9)884-888.

3. Amundsen, M. Guralnick L. And Webster G.D. Variations in strategy for the treatment of urethral obstruction after a pubovaginal sling procedure. J Urol; 2000;164(2)434-437, 2000.

4. Nitti, VW,.Carlson KV, Blaivas JG Dmochowski RR.. Early results of pubovaginal sling lysis by midline sling incision. Urology;2002 59:(1)47-52.

5. Scarpero HM,. Dmochowski RR, Nitti.VW. Repeat urethrolysis after failed urethrolysis for iatrogenic obstruction. $J$ Urol, 169:1013-1016, 2003.

Correspondencia autor: Dra. A. Bujons Tur. Servicio de Urología. Fundació Puigvert. Cartagena, 340-350. 08025 Barcelona. Tel.: 934169700

Email autor: abujons@gmail.com Información artículo: Notaclínica (Trabajo aceptado noviembre 2005) 